\title{
Non-destructive Evaluation of Damage Mechanisms in Composite Sandwich Structure
}

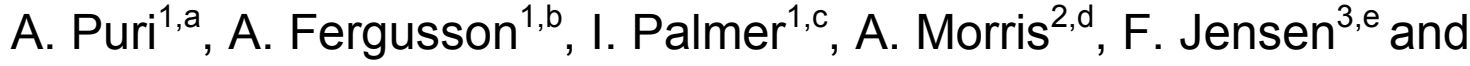 \\ J.P. Dear ${ }^{1, f}$ \\ ${ }^{1}$ Imperial College London, Dept. Mech. Eng. Sth. Kensington, London, SW7 2AZ, UK \\ ${ }^{2}$ E.ON UK, Power Technology, Ratcliffe-on-Soar, Nottingham NG11 0EE, UK \\ ${ }^{3}$ Department of Wind Energy, Risoe National Laboratory at DTU, P.O. Box 49, Roskilde, Denmark \\ aamit.puri01@imperial.ac.uk, ${ }^{\mathrm{b}}$ alexander.fergusson01@imperial.ac.uk, \\ ciain.palmer02@imperial.ac.uk, 'Andy.P.Morris@eon-uk.com, ${ }^{\mathrm{e}}$ find.moehlholt.jensen@risoe.dk, \\ j.dear@imperial.ac.uk
}

Keywords: optical correlation, sandwich structures, bending, failure (mechanical)

\begin{abstract}
This paper presents the experimental results obtained of flexurally loaded wind turbine blade cross section material. All material was extracted from a wind turbine blade box girder and testing was conducted in four point configuration. The aim was to gain an understanding of the structural integrity of this lightweight material as it deforms in flexure. To allow for thorough analysis, digital image correlation (DIC) was used to produce full field strain maps of the deforming specimens. Results highlight the capability of the DIC technique to identify regions of failure, as well as the aspects responsible for them. Overall, the results present a foundation for tests on larger substructure, and eventually integration into manufacturing and maintenance aspects of the industry.
\end{abstract}

\section{Introduction}

The use of lightweight composite materials is an integral factor for aerodynamic applications, such as wind turbine blades. For this application the major benefit is that the inertial loading upon the rotor hub is reduced. Thus, this material is the one primarily used for the blades, and for cost reasons E-glass fibre is the main choice, [1]. Careful control of the lay-up is used to achieve directional strength for the various components. In particular structural foam is used to create sandwich structures that are used for higher flexural stiffness. One of the key challenges with such material is the detection of damage that can occur, and is the main focus of this research.

To simulate failure in this material, specimens were tested in four point configuration conforming the ASTM standard C393-00, [2]. This flexure loading is representative of reality, and to further the simulation, all specimens were extracted from a wind turbine blade box girder reinforcement, which is an internal structural component running the length of the blade to increase the flapwise bending resistance. In addition, to allow for a more thorough examination of failure digital image correlation (DIC) was conducted on the cross section of the specimens.

Presented in this report is a short background on the testing arrangement, followed by a brief description of the DIC technique. The results section follows, giving the output of DIC as a comparison between different sandwich geometries, moving on then to describe the different failures. Following on from this there is a short summary which also discusses the future of the research. 
Testing Arrangement. The specimen geometries and loading configurations are given in Fig. 1. All specimens had widths of $50 \mathrm{~mm}$. This material was extracted from two different regions of the blade, resulting in two different cross-sections. For clarity these have been termed Type 1, which has thick skins and a thin core, and Type 2, which has thinner skins and a thicker core. Both of these types have multiple biaxial $+/-45^{\circ}$ glass fibre layers. The foam used for both specimen types was PVC. All specimens had been machined from larger components which had an element of aging associated with it, but had passed all certification requirements. All tests were conducted at a constant crosshead displacement rate in the range of $0.16 \mathrm{~mm} / \mathrm{s}-8.33 \mathrm{~mm} / \mathrm{s}$.
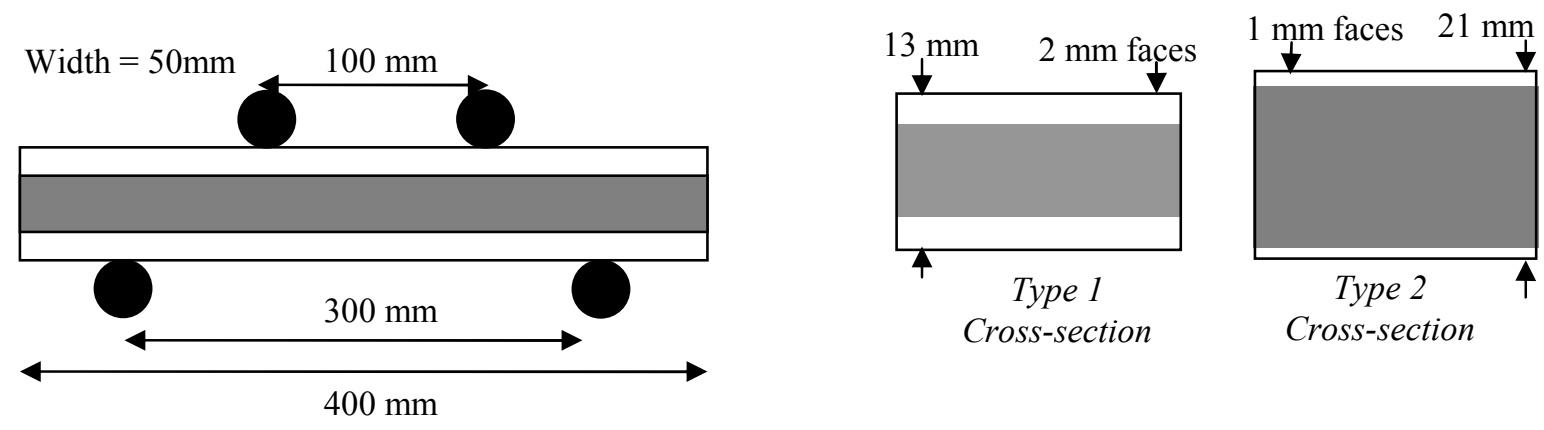

Fig. 1: Sandwich panel loading configuration and cross-section dimensions.

For comparison a layered composite panel was also tested, which does not contain any foam material. This material is used for direct load bearing requirements, such as the bending moment which occurs along the length of the blade. For this the fibres would be primarily aligned along the blade length, as shown below in Fig. 2. Required are some additional biaxial layers to resist the other loading modes applied to the blade, such as torsion. The lay up is again demonstrated in Fig. 2. This material was also extracted from a box girder, and is termed a flange panel. The machined specimens were also $400 \mathrm{~mm}$ long, $50 \mathrm{~mm}$ wide and subjected to the same four point loading as the sandwich material. However, they had a curvature due to aerodynamic profile requirements.

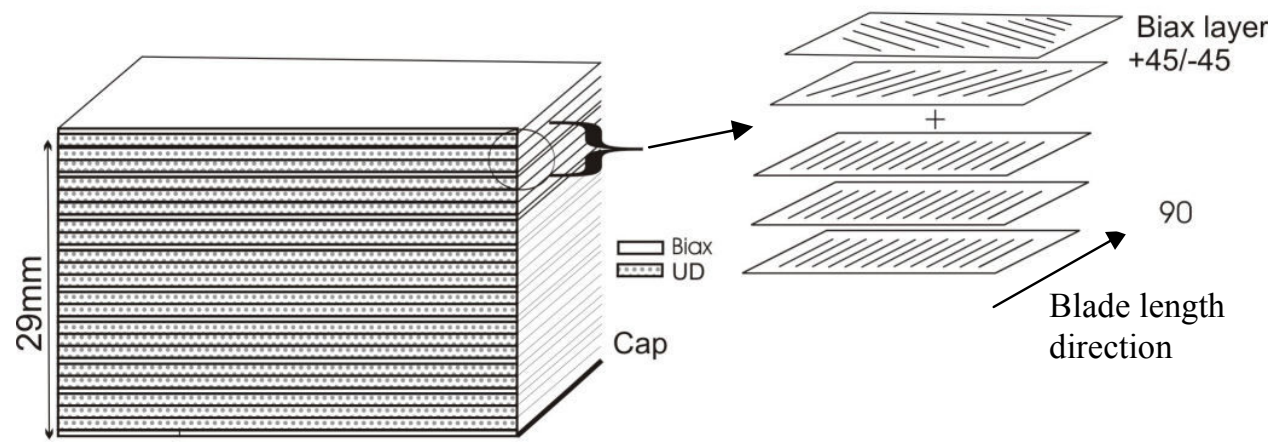

Fig. 2: Flange panel layer arrangement.

Digital Image Correlation. Digital image correlation (DIC) can be used to produce a full-field surface strain map by comparing a series of images captured at various levels of deformations. In order to do this a contrasting monochromatic paint pattern is applied to the component surface, for example a black background with randomly orientated white dots, or vice-versa. The method can be applied without use of a paint pattern so long as there are some distinguishing features. An initial image is captured using a digital camera and is treated as the reference to which all subsequent images are compared. These subsequent images will show the surface paint pattern with some variations as a result of deformation, [3]. In order to achieve a higher degree of accuracy, correlations are based on squares of pixels, known as facets, rather than individual pixel tracing. These facets have an array of greyscale values corresponding to the pattern, thus allowing tracking through the subsequent image stages, [4]. This process is shown in Fig. 3. 


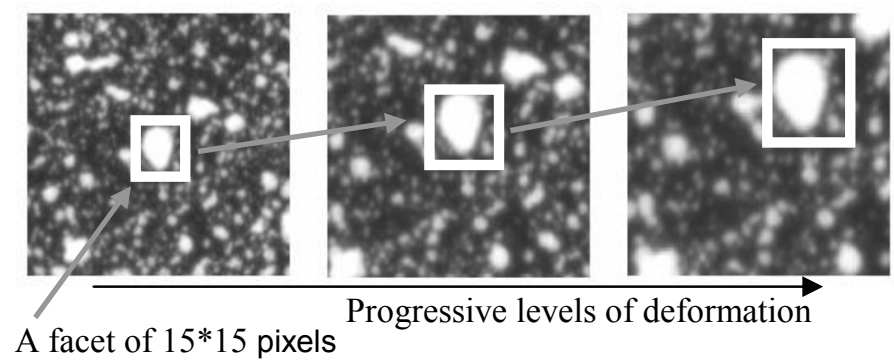

Fig. 3: Example of DIC method.

It is typical to overlap the facets to increase the correlation accuracy, and ensure that no data is missed in the evaluation. The size of the facets and the level of overlap play an important role in determining the correlation, for example strains can be measured in the range from $0.1 \%$ to several $100 \%$ with accuracy of ca. $0.05 \%$ (500 micro-strain) under best conditions, [3]. However, the resolution is related to the useable facet size, which in turn will determine the level of strain variation that can be obtained. For example, if there is a feature in the specimen, then, selecting a facet size which eclipses the complete feature will result in a low strain resolution i.e. the strain pattern associated with the feature will not be visible. However, using larger facets allows more data to be contained within the facet and the accuracy of measured displacement vector associated with the facet is increased.

With an evaluated displacement field the next task for the DIC software is to evaluate the strain. It does this by differentiating the difference between the deformed and undeformed co-ordinates across a number of facets to formulate the displacement gradient tensor for a single facet. The minimum field that can be differentiated across is $3 \times 3$, and it is this field that was used for the experimental results presented in this paper. Using the eight surrounding facets to evaluate a single facet strain leaves an over-determined system, and thus the Gaussian least squares iterative method is employed. The method is employed for all usable facets in the surface. At the edges of a specimen the amount of surrounding facets drops, and if it drops to less than 55\% strain cannot be resolved for that facet, [3].

For these experiments DIC was conducted using a DSLR camera, with images imported into the GOM package ARAMIS 5.4.1, [5]. The higher sensor resolution (10 mega-pixel) of the DSLR allows for facets to represent a smaller physical size than the ARAMIS cameras, however the quality of lens used was inferior to that provided with the GOM system. The result was that there is little difference between the strain results produced by the two camera systems. Yet it was proved that DIC could be made easily transportable by using a DSLR, which would make for easier integration into the wind turbine industry, potentially at this juncture for use during certification tests or studies associated with assessing design integrity. The quoted strain range for this ARAMIS system is $0.05 \%$ to greater than $100 \%$, with an accuracy of $0.02 \%$, although a calibration needs to be run with the DSLR to determine the obtainable accuracy.

\section{Results}

Flange Specimen Testing. Fig. 4 shows the progressive levels of deformation that lead to failure of the flange panel. It can be seen that fracture occurs as a result of high levels of deformation on the tensile side, where the tensile strength of the skin is exceeded and fractures. As the very bottom surface is bi-axial composite it has a higher resistance to failure than the uni-directional (UD) central layers (higher modulus in the horizontal direction because UD fibres are aligned out-ofplane to the image). Thus cracks form in the matrix of the very bottom UD layers, which is better highlighted in Fig. 5. 
To better understand the failure progression the DIC bending strain contour plots have been shown for three load stages in Fig. 6. The images are related to strain via a scale, and the appearance of results can be likened to finite element package output. The first two images have been overlaid onto their respective stage images, but for clarity the final stage does not have this overlay.

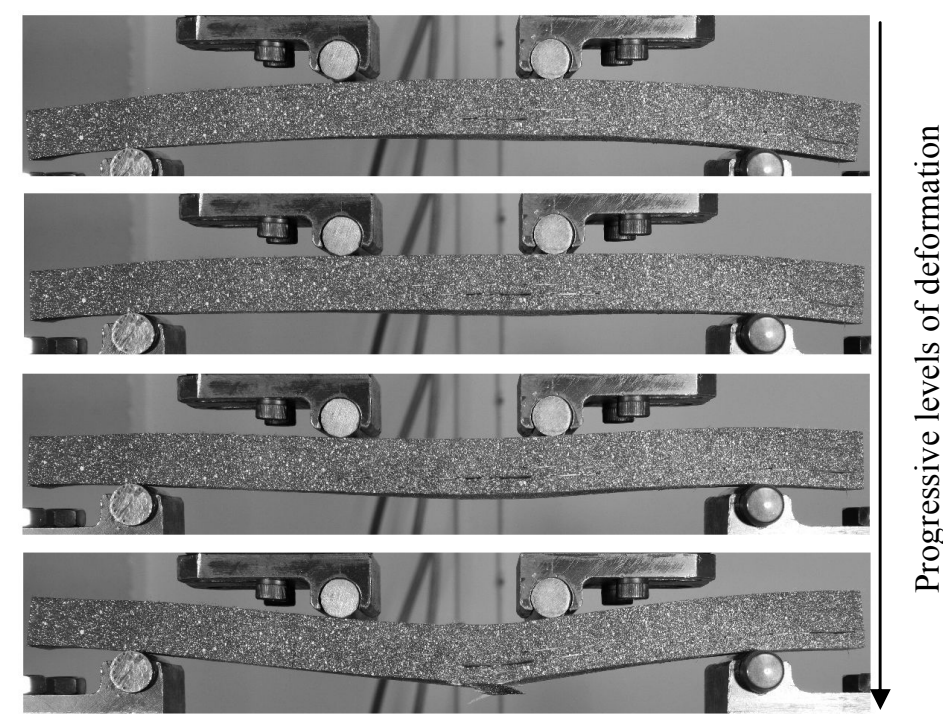

Fig. 4: Failure progression of four point flange specimen.

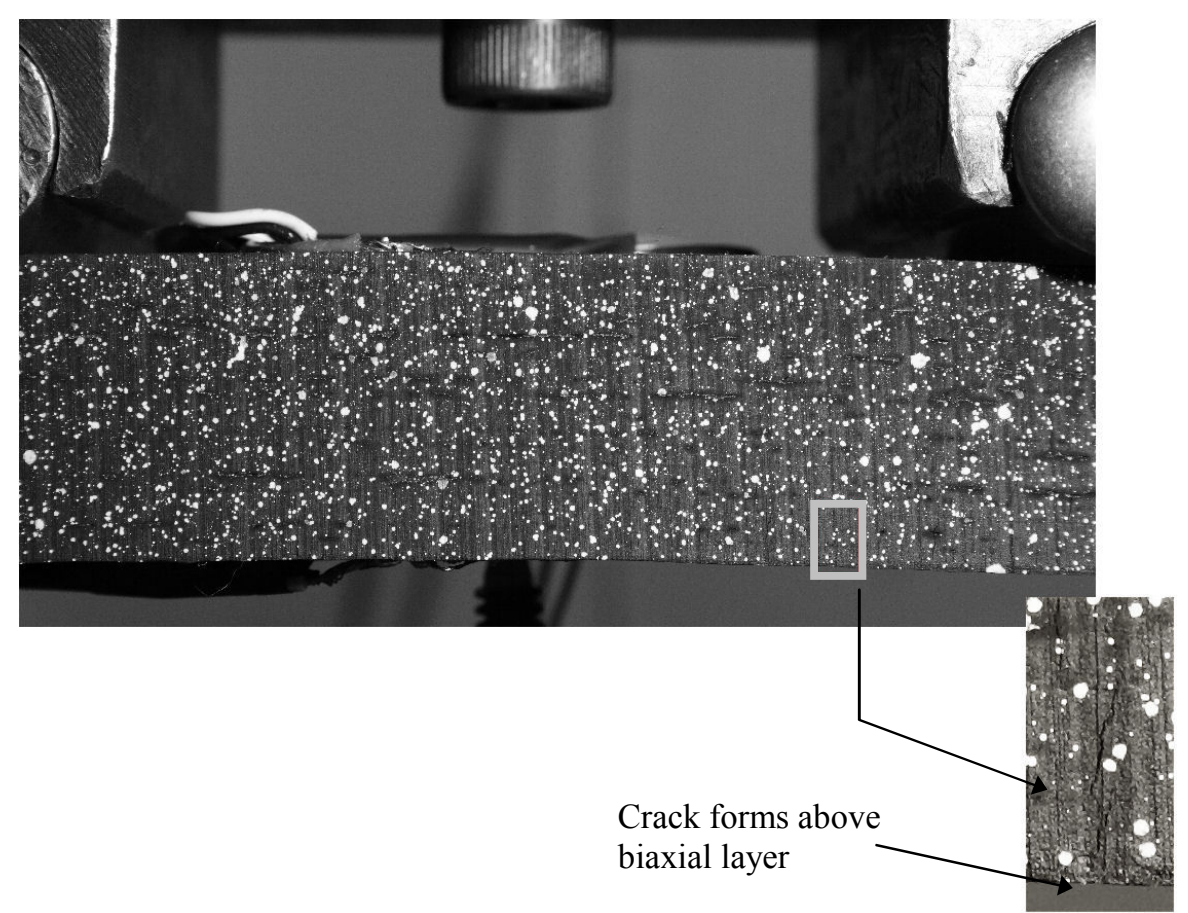

Fig. 5: Crack initiation in flange specimen under four point loading.

The strain here is somewhat confusing as it shows the cracks being under a high level of bending strain, whereas the strain along the discontinuity/crack should be zero. The reason for the high strain is because the pattern on either side of the crack move away from one another, and thus it seems as if the thin crack is straining. Essentially it may be considered that the peak strain contours in this region act to identify the crack position. Higher resolution (pixels per inch) would result in this effect being reduced, and at very high resolution DIC should be able to show zero strain on the crack itself. However, DIC does correctly show zero strain either side of the surface crack, where 
the pattern shouldn't be straining in the $\mathrm{x}$ direction. Thus it has been shown that DIC is able to detect defects as discontinuities in composite layered specimens.

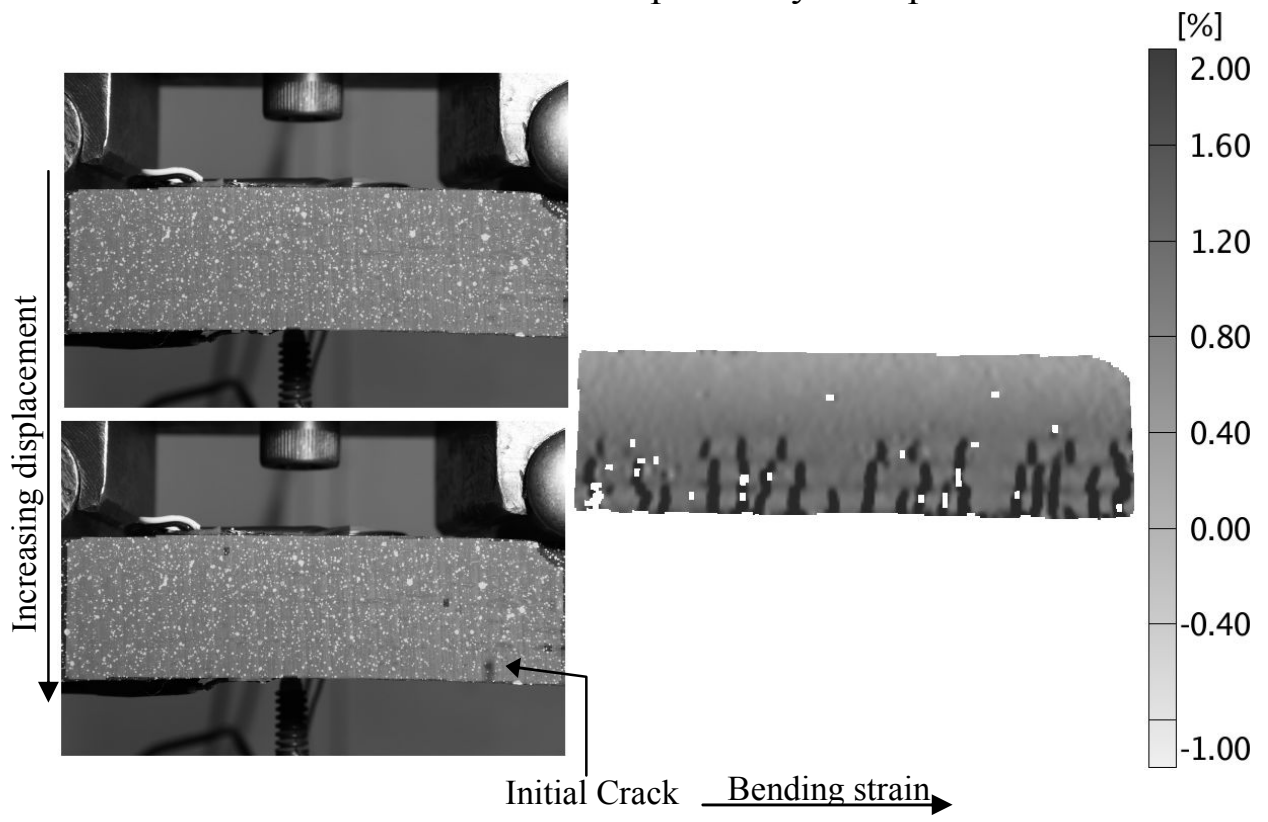

Fig. 6: DIC images highlighting bending strain surrounding cracks.

The overall bending strain distribution is shown more clearly in Fig. 7. As can be seen there is an even distribution at the lower load levels, with compressive behaviour at the top and tensile behaviour at the bottom. Post fracture $(1.7 \mathrm{kN})$ the strain evaluated is highly tensile with some areas being unresolved due to degradation of the pattern. With this camera setup the resolution of strain is reduced, as compared to Fig. 6. However, what is visible is that the characteristic strain variation (compressive to tensile) is maintained.
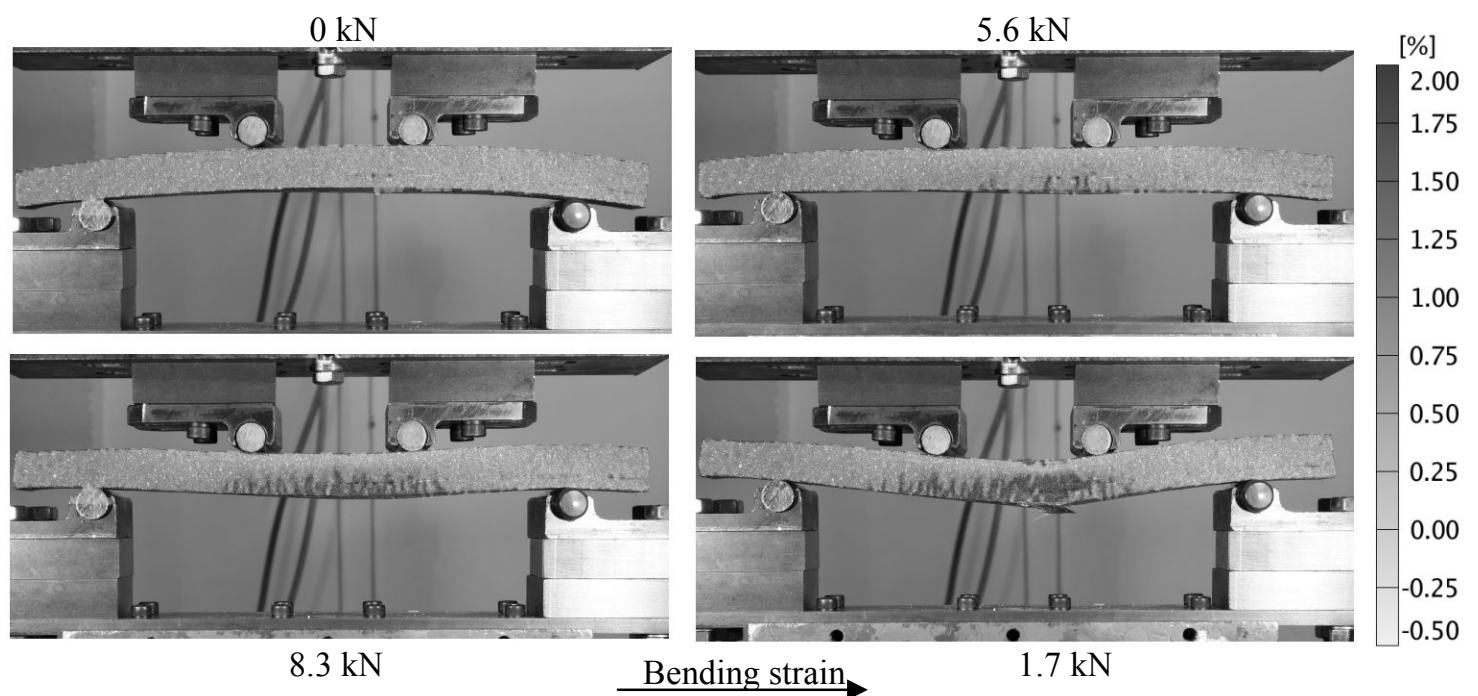

Fig. 7: Flange panel four point bending strain distribution.

Sandwich Specimen Testing. Fig. 8 shows the variation of bending strain for the Type 1 sandwich panel at various levels of deformation. Unlike the results observed for the flange panel, Fig. 7, the strain is high underneath both inner and outer rollers as a result of crushing. This is undesirable, yet between the inner rollers the distribution is clearer, with the strain compressive on the top surface and tensile on the bottom, as expected. In contrast the strain pattern for the Type 2 panel, Fig. 9, shows clearer indentation related strains underneath the inner rollers. This effect is so severe that it masks the behaviour of the strain in between the inner rollers, especially at the higher load levels 


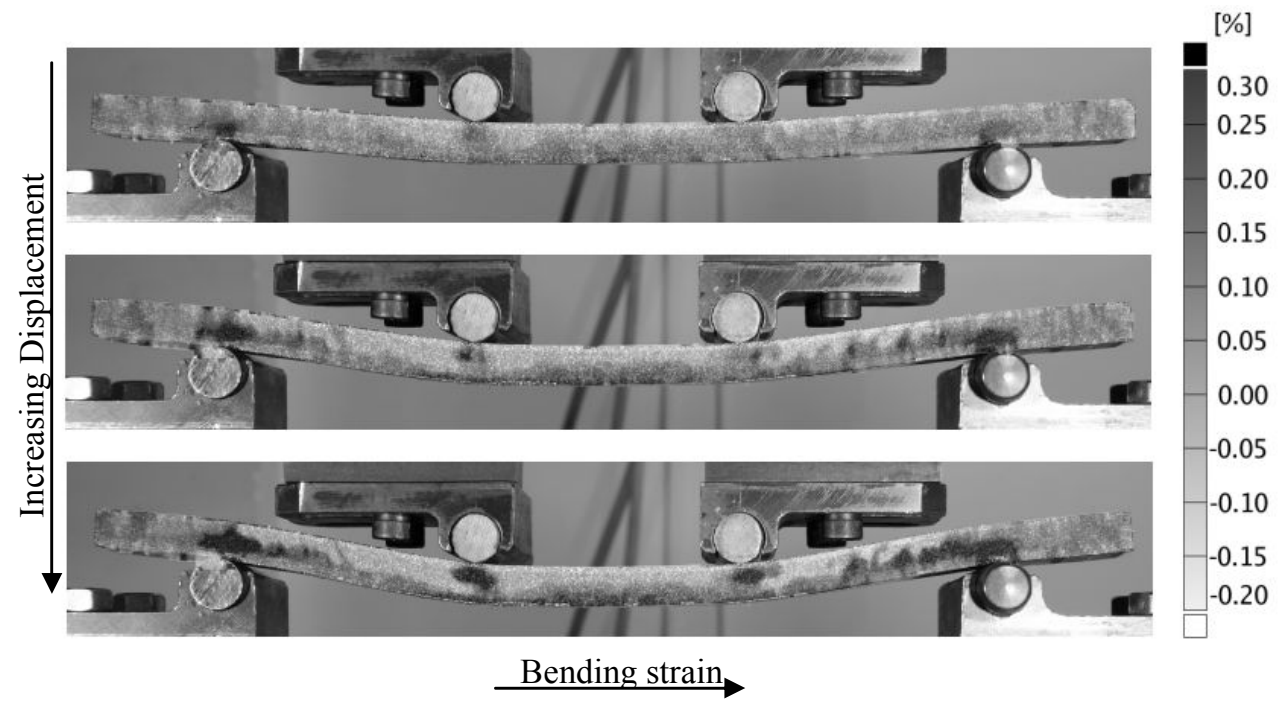

Fig 8: Build-up of bending strain for type 1 sandwich panel.

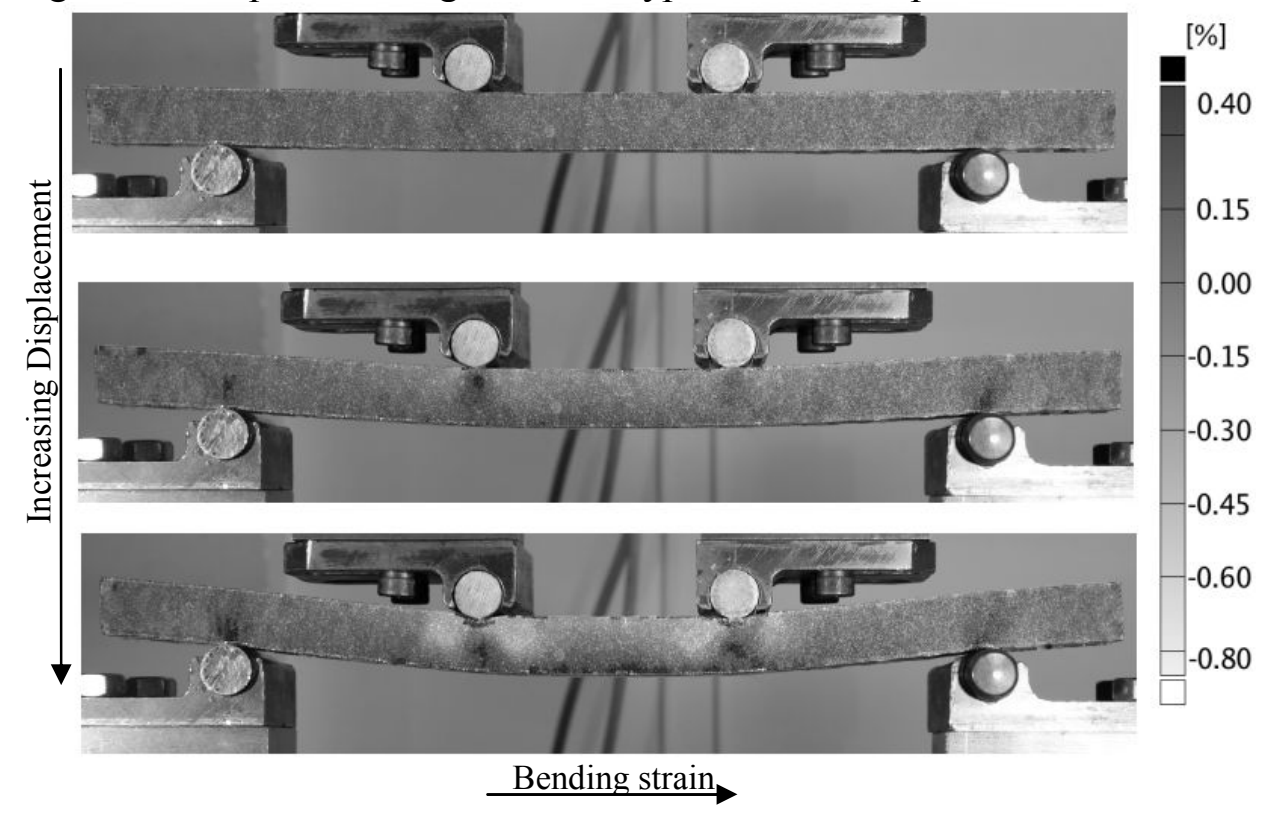

Fig 9: Build-up of bending strain for type 1 sandwich panel.

To quantify this analysis Fig. 10 shows the difference between the bending strain distributions for the two panel types along the specimen centreline at low and high levels of crosshead displacement. The low crosshead displacement graph shows that dissimilar levels of strain are observed when the two panels are under pure flexure. The Type 1 panel is showing a more conventional bending strain distribution, with the neutral axis close to halfway through the specimen. However, the Type 2 panel shows a significant amount of distortion along the profile. A clearer result is that of the intermediate displacement, which shows bending strain at peak load for Type 2. At this stage both specimens are just leaving pure flexure, and the result is symbolic of the higher flexural rigidity of the Type 2 panel, i.e. the Type 2 panel has a higher load at this displacement compared to Type 1, and hence greater strain. It can be seen that the bending strain is more closely matched at high levels of crosshead displacement, even though the two specimens are in states of failure via different mechanisms. A possible reason for this behaviour is because the Type 2 panel is entering the secondary stage where densification means that the foam is providing increasing resistance to further indentation. The two different mechanisms of failure are discussed next. 


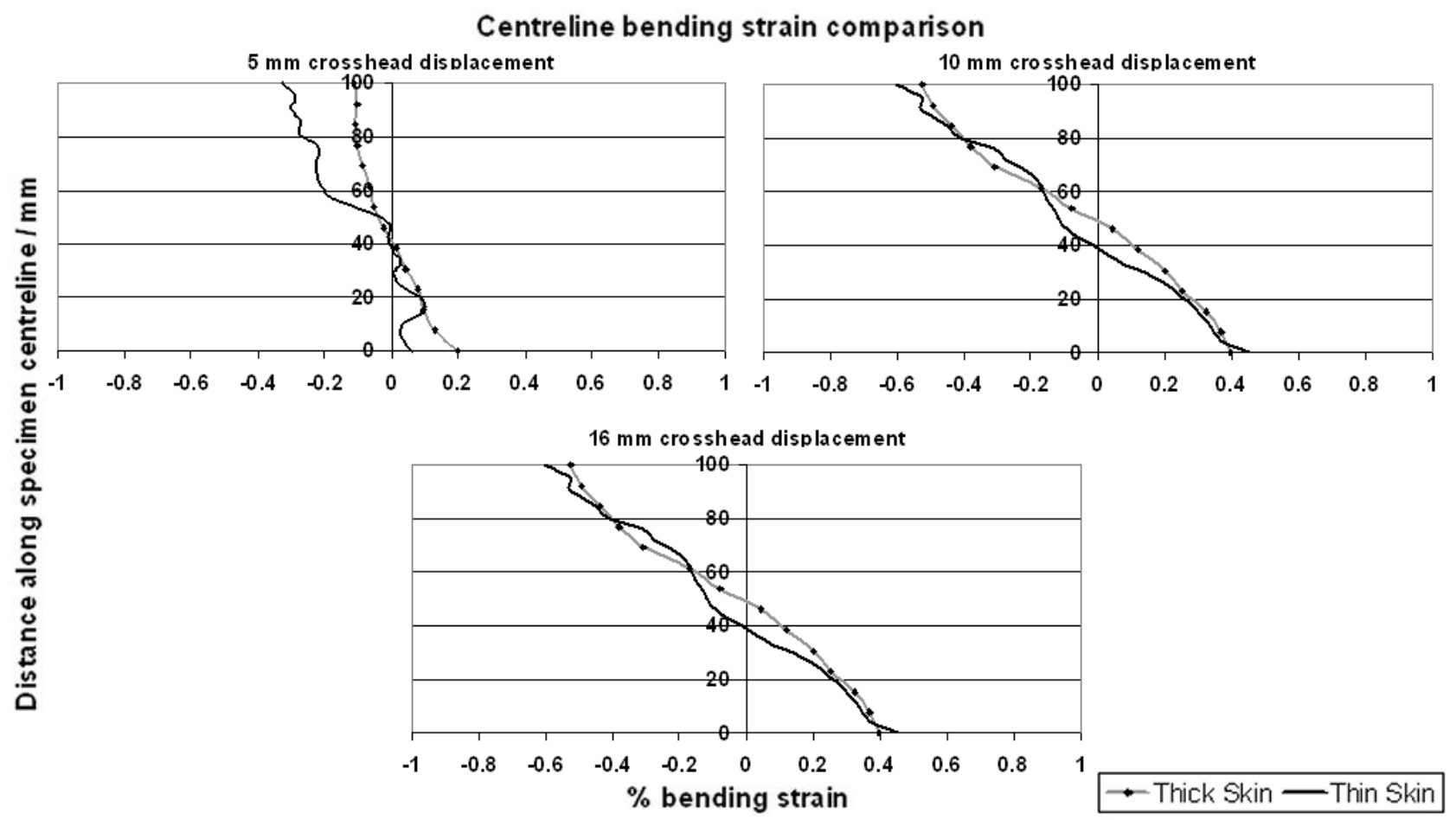

Fig 10: Comparison of specimen centreline bending strain at low and high crosshead displacement for two sandwich panels.

Failure Mechanisms. Although indentation failure is undesirable, it does allow for an examination of the DIC techniques applicability to this type of material. The onset of indentation is better highlighted in Fig. 11, which is displaying the DIC evaluated vertical displacement of the top, middle and bottom of the Type 2 sandwich panel for increasing crosshead displacement. These graphs are shown for the left and right roller, as well as the centre of the specimen.

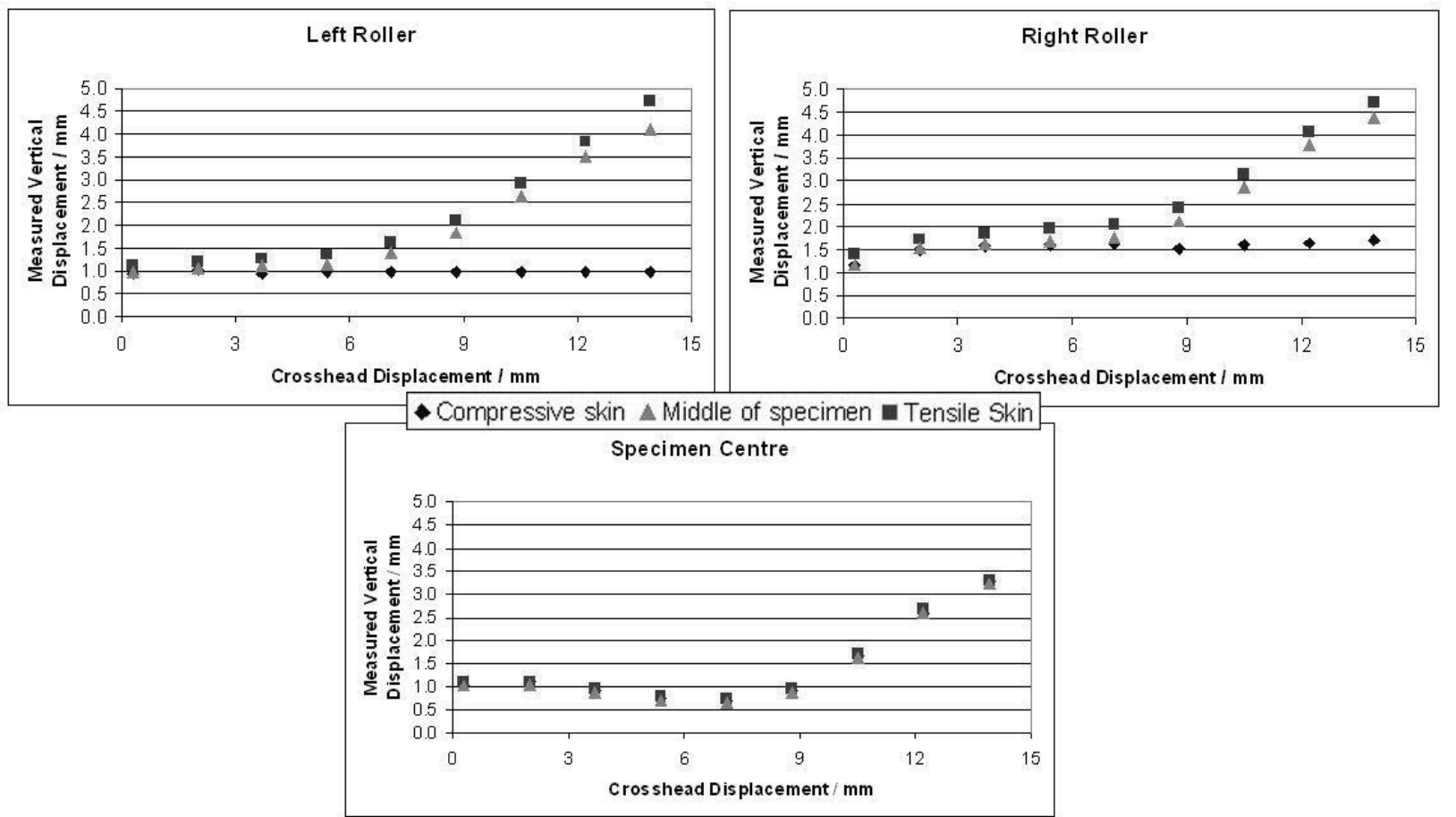

Fig 11: Vertical displacement plotted against crosshead displacement for left and right inner rollers, and specimen centre. 
What is evident is that at the two rollers the compressive skin (top) is essentially pinned to the inner rollers, which are stationary whilst the outer rollers move upward. However, the bottom and middle sections do show vertical motion upward. This means that the middle and bottom of the specimen squash into the top section at the roller location. This behaviour is not observed with the centre of the specimen and instead the three elements stay together at all values of crosshead displacement, and effectively the level of indentation can be quantified. An additional observation is that the middle of the specimen is showing similar levels of indentation as the bottom, which indicates that crushing is more severe in the compressive side of the specimen than the tensile side, which is an effect that was visually observed.
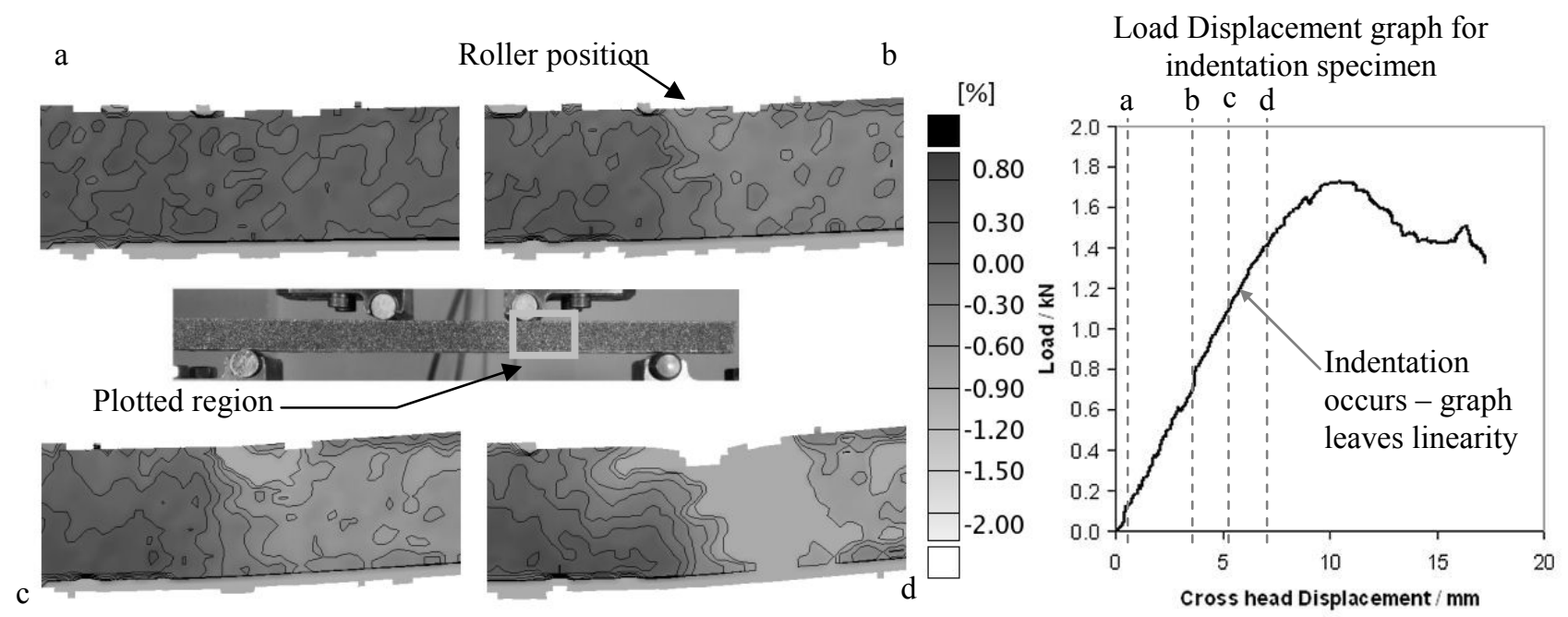

Fig 12: Close up of DIC $\varepsilon_{2}$ strain at increasing percentages of indentation load: a) $8 \%$; b) $66 \%$; c) $92 \%$; d) $115 \%$. Supporting load displacement graph on right hand side.

The DIC results along the indentation path are shown in Fig. 12. Plotted here is the principle strain $\varepsilon_{2}$ for increasing load levels, expressed as a percentage of the load causing initial indentation. This indentation load has been calculated using the load-displacement plot shown in the supporting graph, where indentation causes the first deviation from the linear loading response. This load was cross-verified against DIC evaluated displacement recorded at the roller sites, i.e. Fig. 11.

In Fig. 12 the initial stage reveals no particular strain distributions or features. By the second stage ( $66 \%$ of the indentation load) the effects become detectable as a clear differentiation between the two sides where on the left of the roller the majority of strain is representative of bending, and on the right hand side where the strain is due to shear. At the higher loads the strain contours are more representative of indentation, with high levels of compression occurring. Thus DIC could be used to estimate the strain level that would result in initial crushing of the core, which for this foam was $2.3 \%$.

The Type 1 sandwich panel did not fail via indentation, but rather by core shear. Here the shear in the core is at a high level, causing collapse of some cell walls along a $45^{\circ}$ line close to the inner roller, in-between the inner and outer rollers. This region is like a kink in the core that results in the departure from pure bending of the specimen, resulting in a plateau of the load levels.

A related failure was obtained on Type 1 specimen by using smaller diameter inner rollers, which resulted in some indentation. Here shear failure and eventually a crack did develop for this specimen, as shown in Fig. 13a. This figure also displays how the compressive skin hinges at the inner rollers, which is required to compensate for the core shear failure (otherwise the compressive skin in between the rollers would have some greater curvature), [6].

The DIC strain plots for this failure are shown in Fig. 13b, where shear angle has been plotted. What is shown here is that indentation initially pins the compressive skin to the inner roller, and deformation has to occur as a result of shear in the adhesive layer between foam core and 
compressive skin. This is highlighted clearly in this the second stage of this image series. After further deformation the shear of the adhesive is excessive, and it is believed that a disbond between the compressive skin and core triggers a fracture of the core, as shown in the third image stage. This result is particularly relevant to wind turbine loading, as compared to indentation which is unlikely to occur.
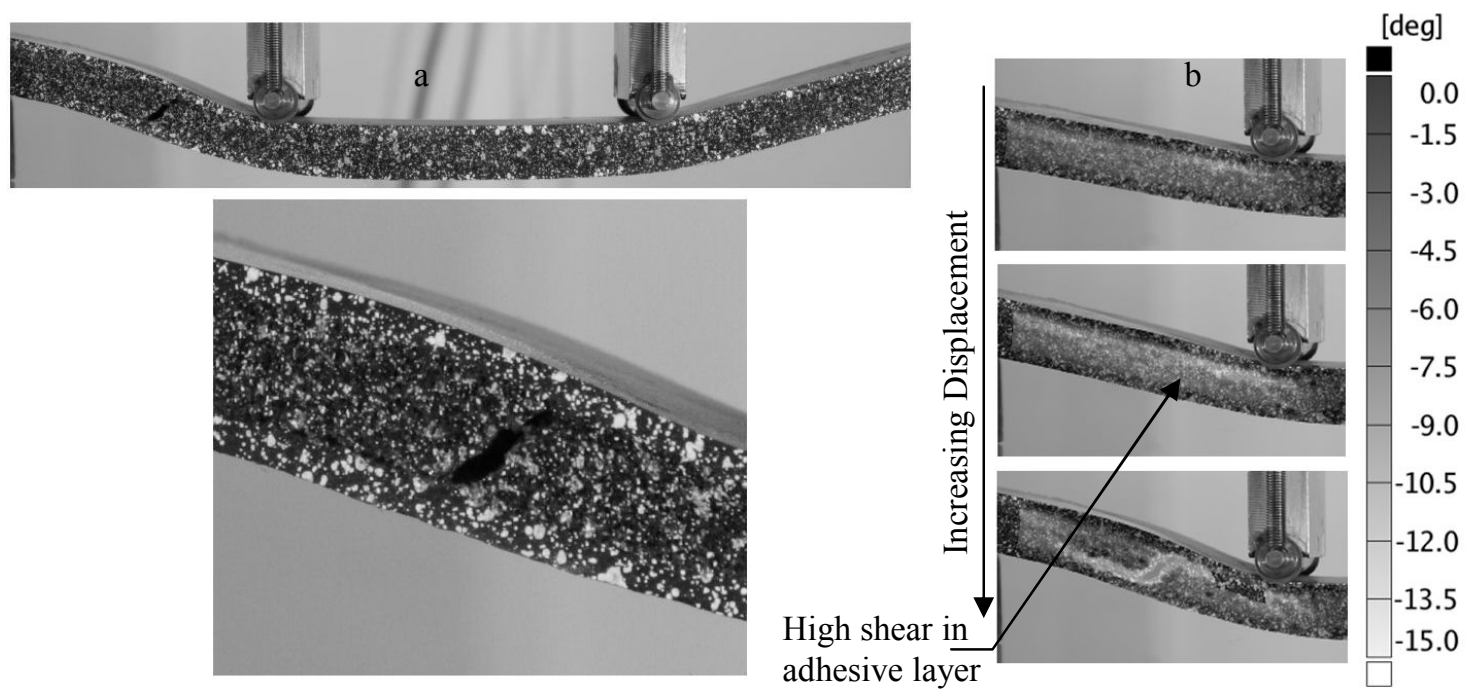

Fig 13: a) Core fracture of type 1 sandwich specimen; b) Core shear failure DIC sequence plot (shear angle).

\section{Conclusions}

One aim of this research was to gain a better insight into the failures that occur in lightweight sandwich material as a result of flexural loading. However, the failures processes observed were affected by the specimen shape and loading configuration. For example, the flange panels did show a relevant failure path, but the initiation of this failure was a result of surface cracks. This is unlikely to occur in reality because the component does not generally have free surfaces, [7]. Regardless of this, there was good demonstration that DIC was able to identify the relevant subsequent failure progression. For future relevance, it seems likely that sandwich material will be considered for use in the flange as well as webs, [8]. From this it seems as if greater concentration on sandwich material testing is needed, which didn't seem to suffer from surface effects in the same way as the composite flanges.

In a similar manner, the Type 2 sandwich panel failed via indentation, something which holds little relevance, but again DIC proved a useful tool in the understanding of this failure. With a calibrated setup, preferably using 3D capture, the methods described could benefit manufacturers design assessments. For example, a new geometry could be tested for structural integrity prior to major rollout of the design.

In addition to this, the experimental results for the Type 1 sandwich panel showed a representative failure mode with the high levels of shear in the adhesive and core. The capability of DIC to recognise relevant failures at load levels prior to non-reversible failure was demonstrated. This result has implications for inspection and maintenance of blades, where the technique could be used to flag up regions requiring attention on an aged or defected blade. To further this suggestion, DIC needs to be proved to be able to detect sub-surface defects, such as delaminations.

However, DIC is not the only suitable method for NDE of composite material. The majority of DIC results presented are comparing one region of strain to another, and effectively the actual values of strain are not being used. Such a comparison can be made more easily with other techniques, for example shearography or Moiré Fringe interferometry, [9, 10]. Yet this experimental work is at an early stage, and it is predicted that the strain data will serve a multitude of functions, ranging from a quantifiable measure of damage, direct comparison work with FE designs and a 
unique way of determining the severity of subsurface defects. At this early experimental stage it also allows a comparison against standard experimental equipment such as displacement transducers and strain gauges, comparisons which have been made, but are not presented here for conciseness.

In summary, the test results presented show the versatility of the DIC system, and present a stepping stone for tests on larger substructure. At this stage, it is believed that the technique could benefit blade development and manufacturing, as well as inspection and maintenance. Future experiments will develop upon the testing procedures shown, as well as introducing other aspects such as acoustic emissions (AE) monitoring, which has been receiving a lot of attention from research groups experimenting in the wind turbine field recently, [11-13]. What has been demonstrated generally is that $\mathrm{AE}$ is capable of giving early indication of some damage in a composite structure, with potentially some indication of the type of damage incurred. It is envisaged that on a wind turbine blade the DIC system could be used to identify the exact location and extent of the damage. This information could then be fed back into an FE model to allow lifetime predictions, or even to a maintenance team who could target their repair strategy. The overall aim of this research is provide a toolbox of complementary proven techniques and strategies that can give wind turbine owners confidence about the structural integrity of their ageing blades.

\section{References}

[1] DNV and RISO: Guidelines for Design of Wind Turbines, Scanprint, Denmark, (2002)

[2] ASTM International: ASTM C393 Standard Test Method for Core Shear Properties of

Sandwich Constructions by Beam Flexure, (2000)

[3] GOM mbH: ARAMIS user manual, GOM mbH, (2005)

[4] M. Sjodahl: Accuracy in electronic speckle photography, Applied Optics, 36 (1997), 2875-85

[5] GOM mbH: ARAMIS, http://www.gom.com/EN/measuring.systems/aramis/system/system.html,

[6] J. B. Sha, T. H. Yip and S. K. M. Wong: In situ surface displacement analysis of fracture and fatigue behaviour under bending conditions of sandwich beam consisting of aluminium foam core and metallic face sheets, Materials Science and Technology, 22 (2006), 51-60

[7] B. F. Sorensen, E. Jorgensen, C. P. Debel, F. M. Jensen, H. M. Jensen, T. K. Jacobsen and K. M. Halling: Improved Design of Large Wind Turbine Blades of Fibre Composites Based on Studies of Scale Effects (Phase 1) - Summary Report, Riso Ntional Laboratory, (2004)

[8] C. Berggreen, K. Branner, J. F. Jensen and J. P. Schultz: Application and analysis of sandwich elements in the primary structure of large wind turbine blades, Journal of Sandwich Structures and Materials, 9 (2007), 525-552

[9] H. Sutherland, A. Beattie, B. Hansche, W. Musial and J. Allread: Application of nondestructive techniques to the testing of a wind turbine blade, (1994)

[10]F. M. Jensen and A. Morris: Full-Scale Testing and Finite Element Simulation of a 34m Long Wind Turbine Blade, Dept. of Wind Energy, Risoe National Laboratory, DTU,

[11] M. J. Blanch and A. G. Dutton: Acoustic emission monitoring of field tests of an operating wind turbine, Key Engineering Materials, 245-346 (2003), 475-480

[12]P. A. Joosse, M. J. Blanch, A. G. Dutton, D. A. Kouroussis, T. P. Philippidis and P. S. Vionis: Acoustic emission monitoring of small wind turbine blades, Journal of Solar Energy Engineering, Transactions of the ASME, 124 (2002), 446-454

[13] A. G. Beattie: Acoustic emission monitoring of a wind turbine blade during a fatigue test, (1997) 